\title{
Role of G protein-coupled receptors (GPCRs) for purines and pyrimidines in mediating degeneration and regeneration under neuroinflammatory processes
}

\author{
Heike Franke
}

Published online: 18 March 2011

(C) Springer Science+Business Media B.V. 2011

\section{Part I. Presented by Maria P. Abbracchio}

Peterson TS, Camden JM, Wang Y, Seye CI, Wood WG, Sun GY, Erb L, Petris MJ, Weisman GA (2010) P2Y 2 Nucleotide Receptor-Mediated Responses in Brain Cells. Mol Neurobiol 41:356-366; DOI 10.1007/s12035-010-8115-7

\section{Article summary}

G.A. Weisman and his co-authors summarize and discuss a number of remarkable own findings and data from other groups relating to the mechanisms underlying the proinflammatory and neuroprotective effects mediated by the $\mathrm{G}_{\mathrm{q}}$ protein-coupled $\mathrm{P} 2 \mathrm{Y}_{2}$ receptor $\left(\mathrm{P} 2 \mathrm{Y}_{2} \mathrm{R}\right)$ subtype in glial cells and neurons and their potential relationship to the pathophysiology of Alzheimer's disease (AD).

The main hypothesis of the present review is that chronic stress or damage of the brain causes an increased release of nucleotides resulting in the activation of $\mathrm{P}_{2} \mathrm{Y}_{2}$ Rs. This leads to the induction of pro-inflammatory responses in glial cells with subsequent upregulation of neuronal $\mathrm{P} 2 \mathrm{Y}_{2} \mathrm{Rs}$, which in turn mediates neuroprotective effects.

Previous studies have shown that in $\mathrm{AD}$, the level of the proinflammatory cytokine interleukin-1 $\beta$ (IL-1 $\beta$ ) is elevated, in part due to nucleotide stimulated release from glial cells. Oligomeric $\beta$-amyloid peptide ( $A \beta_{1-42}$ ), widely accepted as a contributor to neurodegeneration in $\mathrm{AD}$, increases nucleotide release from astrocytes, which would serve to activate $\mathrm{P} 2 \mathrm{Y}_{2} \mathrm{Rs}$ in neurons. Furthermore, the stimulation of rat primary cortical cultures with IL-1 $\beta$ induces an increased $P 2 \mathrm{Y}_{2} \mathrm{R}$ expression in

\section{H. Franke $(\bowtie)$}

Rudolf Boehm Institute of Pharmacology and Toxicology,

University of Leipzig,

Leipzig, Germany

e-mail: Heike.Franke@medizin.uni-leipzig.de neurons. Finally, activation of $\mathrm{P}_{2} \mathrm{Y}_{2}$ Rs with UTP appeared neuroprotective, since this enhances the non-amyloidogenic cleavage of amyloid precursor protein (APP).

The consequences of the $\mathrm{P} 2 \mathrm{Y}_{2} \mathrm{R}$ activation are summarized in detail with a clear differentiation between astrocytes (heterologous expression of $\mathrm{P}_{2} \mathrm{Y}_{2} \mathrm{R}$ in human $1321 \mathrm{~N} 1$ astrocytoma cells) and neurons (rat primary cortical neurons).

When expressed in glial cells, the $\mathrm{P}_{2} \mathrm{Y}_{2} \mathrm{R}$ subtype regulates a variety of signal transduction pathways via

1. Phospholipase C (PLC), activating different second messenger pathways (e.g. PKC, PLA 2 , ERK1/2);

2. Phosphorylation of the epidermal growth factor receptor (EGFR). This process depends on the direct interaction of an Src homology binding sequence in the intracellular C-terminal domain of the $\mathrm{P} 2 \mathrm{Y}_{2} \mathrm{R}$, promoting the transactivation of EGFR and the regulation of some key functions, such as, for example, the proliferation of cortical astrocytes.

3. Activation of $\alpha_{V} \beta_{3 / 5}$ integrins that bind directly to an Arg-Gly-Asp (RGD) motif in the first extracellular loop of the receptor subtype, mediating cytoskeletal rearrangements and increasing astrocyte migration-finally associated with reactive astrogliosis in the AD brains.

Interestingly, the latter two effects $(2,3)$ seem to be G-protein independent and highlight a rather new way of signalling of GPCRs that does not involve coupling to G-proteins but depends on some intrinsic specific structural features of GPCRs.

In neurons, the treatment with IL-1 $\beta$ induces a $\mathrm{P} 2 \mathrm{Y}_{2} \mathrm{R}$ upregulation, which promotes

4. Proteolytic processing of non-amyloidogenic cleavage of APP, which precludes the formation of $A \beta$;

5. Phosphorylation of the cytoskeletal protein cofilin, which stabilizes neurite outgrowth and dendritic spines-finally resulting in neuroprotective responses in $\mathrm{AD}$. 
In summary, all these data suggest that $\mathrm{P} 2 \mathrm{Y}_{2} \mathrm{Rs}$ represent a very interesting pharmacological target in neurodegenerative and pro-inflammatory diseases.

The complexity of these effects has been very elegantly summarized by the authors in one of their figures (see Fig. 1 reproduced from Fig. 2 of the Peterson's et al. paper), where, in particular, the pathways highlighted by the red arrows refer to some of the $\mathrm{G}$ protein-independent effects mediated by $\mathrm{P}_{2} \mathrm{Y}_{2} \mathrm{Rs}$.

\section{Commentary}

The aetiology of AD includes the excessive deposition of the neurotoxic $A \beta$ peptide in neuritic plaques, the formation of intracellular neurofibrillary tangles and the neuronal degeneration across multiple transmitter systems. Furthermore, astrogliosis is a well-known feature of AD. The present review highlights a number of important interactions with focus on the $G_{q}$ protein-coupled $P 2 Y_{2} R$ and its involvement in neuroinflammatory processes. A number of studies have shown that inflammation begins as a neuroprotective mechanism (e.g. clearance of the neurotoxic soluble $\mathrm{A} \beta$ peptide) but becomes neurodegenerative when inappropriately sustained, finally resulting in the loss of brain function in AD. Increased ATP release during injury can enhance the inflammatory effects of cytokines and may contribute to the chronic inflammation e.g. seen in AD. The uridine nucleotide-activated $P 2 Y_{2} R$ subtype, a member of the family of eight $G$ protein-coupled P2YRs ( $\mathrm{P} 2 \mathrm{Y}_{1,2,4,6,11-14}$ ), is known to be expressed in diverse tissue types in peripheral and central nervous system, mediating multiple physiological functions. The authors highlight in the summary the activation of the $\mathrm{P} 2 \mathrm{Y}_{2} \mathrm{R}$ subtype as "signalling complex" mediating trophic and neuroprotective interactions of neurons and astrocytes.

Agonist (ATP, UTP)-induced activation of $\mathrm{P} 2 \mathrm{Y}_{2} \mathrm{Rs}$, expressed in glial cells, stimulates the integrin/Rho kinasepromoted glial cell migration, influencing chemotactic activity as well as transactivation of e.g. EGFRs. Following stimulation of mitogen-activated protein kinases (MAPK, ERK1/2) the regulation of gene transcription resulted e.g. in

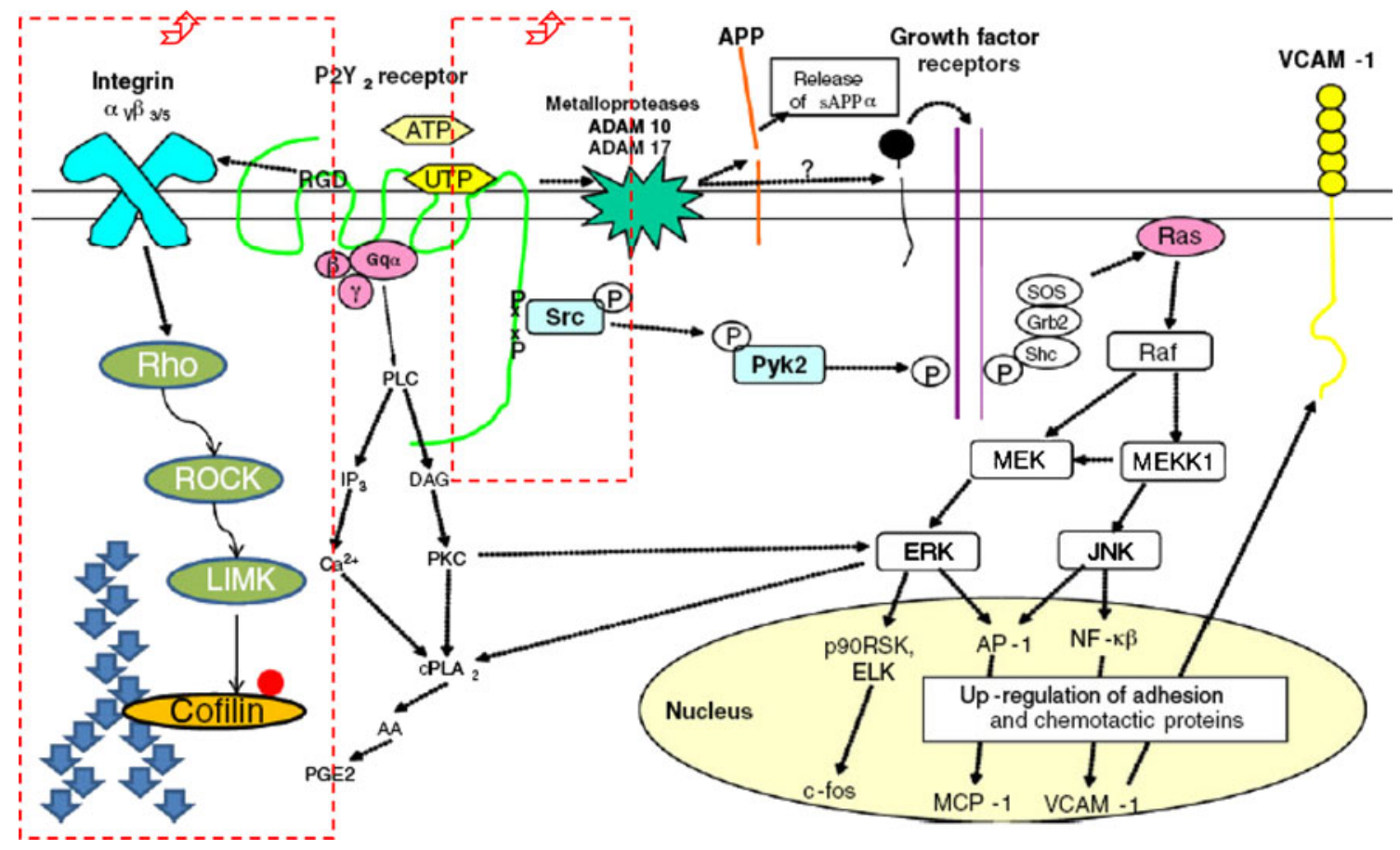

Fig. 1 Figure reproduced with kind permission from Springer Science+Business Media: Molecular Neurobiology, P2 $Y_{2}$ Nucleotide Receptor-Mediated Responses in Brain Cells, Volume 41, 2010, pages 356-366, Troy S. Peterson, Jean M. Camden, Yanfang Wang, Cheikh I. Seye and W. G. Wood, et al., Figure 2. P2Y $\mathrm{Y}_{2}$ receptormediated signal transduction: activation of the $\mathrm{P} 2 \mathrm{Y}_{2}$ receptor $\left(\mathrm{P} 2 \mathrm{Y}_{2} \mathrm{R}\right)$ is coupled to several intracellular signal transduction pathways including: (a) $\mathrm{Gq} \leftarrow$ dependent activation of phospholipase $\mathrm{C}$ (PLC) that generates inositol 1,4,5 trisphosphate (IP3) and diacylglycerol (DAG), second messenger for intracellular calcium mobilization and protein kinase $\mathrm{C}$ activation, respectively; (b) Srcmediated transactivation of growth factor receptor phosphorylation that stimulates mitogen-activated protein kinase cascades to regulate gene transcription; (c) association with and activation of $\alpha v \beta 3 / 5$ integrins that stimulates Rho kinase leading to cofilin phosphorylation; and (d) activation of metalloproteases (i.e., ADAM10/17) to stimulate the non-amyloidogenic processing of amyloid precursor protein (APP). Other abbreviations: AA arachidonic acid, PGE2 prostaglandin E2, VCAM-1 vascular cell adhesion molecule-1 
astroglia proliferation. Furthermore, also the activation of the p38 signalling pathway may be a consequence of $\mathrm{P}_{2} \mathrm{Y}_{2} \mathrm{R}$ stimulation. In this context, the role of EGFR signalling in the regulation of neuronal survival by promoting apoptosis as endpoint of the receptor activation is discussed. Of particular interest is the issue that ATP and UTP (in their interaction with the "sensor" $\mathrm{P} 2 \mathrm{Y}_{2} \mathrm{R}$ ) have been identified as "critical find-me signals" important for phagocyte chemoattraction by apoptotic cells (Elliott et al. 2009, Nature, 461: 282-286). Also, the involvement of $\mathrm{P}_{2} \mathrm{Y}_{2} \mathrm{Rs}$ in microglia in inflammatory processes was emphasized. Actual data using tissue slices indicated that the activation of e.g. $\mathrm{P} 2 \mathrm{Y}_{2} \mathrm{Rs}$ with UTP results in an inflammatory response, measured as a rapid rise in intracellular $\mathrm{Ca}^{2+}$ and release of cytokines (Lee et al. 2011, Brain Res, 1369: 21-35).

One of the highlights of the present data is the cell typespecific upregulation of the $\mathrm{P}_{2} \mathrm{Y}_{2} \mathrm{R}$ and its involvement in neuroprotective processes under inflammatory conditions. APP, normally involved in synaptic formation and repair, is proteolytically cleaved by secretases to the neurotoxic $A \beta$ or alternatively to the non-amyloidogenic peptide soluble amyloid precursor- $\alpha$ (sAPP $\alpha)$. P2 $\mathrm{Y}_{2} \mathrm{R}$ activation stimulates the $\alpha$ - and $\gamma$-secretase-dependent proteolytic processing of APP to generate $\operatorname{sAPP} \alpha$, which has been shown to have neurotrophic and neuroprotective properties. Additionally, upon cofilin phosphorylation, the $\mathrm{P}_{2} \mathrm{Y}_{2} \mathrm{R}$ induces actin cytoskeletal rearrangements, which promote dendritic spine growth and stabilization in neurons. Indeed, these are two evidences supporting the hypothesis that inflammatory upregulation of $\mathrm{P}_{2} \mathrm{Y}_{2} \mathrm{Rs}$ in neurons may be beneficial.

However, the status of $\mathrm{P} 2 \mathrm{Y}_{2} \mathrm{R}$ in human $\mathrm{AD}$ brain is at present nearly unclear. A possible role of $\mathrm{P} 2 \mathrm{Y}_{2} \mathrm{R}$ alterations in regulating molecular processes underlying plaque and tangle formation was investigated using postmortem materials from a cohort of longitudinally assessed $\mathrm{AD}$ patients and aged controls (Lai et al. 2008, J Neural Transm, 115:11651172). $\mathrm{P} 2 \mathrm{Y}_{2}$ immunoreactivity was found to be selectively reduced in the $\mathrm{AD}$ parietal cortex, correlating with neuritic plaque, neurofibrillary tangle scores (high ratings) and synapse loss. In relation to the role of $\mathrm{P}_{2} \mathrm{Y}_{2} \mathrm{R}$ in the nonamyloidogenic pathway, e.g. in the initial stages of neuroinflammation, the observed loss suggests a dysfunction of the P2YR-mediated trophic support in neuronal regeneration/ differentiation (Arthur et al. 2005, Proc Natl Acad Sci USA, 102:19138-19143) or may be a consequence of changed vulnerability of the cells and neuronal cell degeneration in the later, chronic stages of the neuroinflammation in $\mathrm{AD}$ brains. The first specific association between $\mathrm{P} 2 \mathrm{Y}_{2} \mathrm{R}$ expression and neuropathological features in human tissue is given, but further studies are required to answer more detailed questions. Furthermore, selective $\mathrm{P} 2 \mathrm{Y}_{2} \mathrm{R}$ ligands as potential candidates for $\mathrm{AD}$ therapy are needed to improve our basic orientations concerning neurodegenerative diseases.
Finally, in addition to changes of the $\mathrm{P}_{2} \mathrm{Y}_{2} \mathrm{R}$ expression in $\mathrm{AD}$, alterations of the $\mathrm{P} 2 \mathrm{Y}_{1} \mathrm{R}$ subtype (Moore et al. 2000, Neuroreport, 11:3799-3803) as well as the prominent role of the P2X7R subtype (e.g. Parvathenani et al. 2003, J Biol Chem, 278:13309-13317) should be mentioned. Extracellular ATP, acting through the P2X7R, can alter $\mathrm{A} \beta$-induced cytokine release from macrophages and microglia and makes this subtype to an important modulator of neuroinflammation in AD (Rampe et al. 2004, J Neuroimmunol, 147:56-61; Sanz et al. 2009, J Immunol, 182:4378-4385).

In summary, the presented paper improves our understanding of how $\mathrm{P} 2 \mathrm{Y}_{2} \mathrm{Rs}$ work under inflammatory conditions. The data obtained so far provide a good basis for considering $\mathrm{P}_{2} \mathrm{Y}_{2} \mathrm{R}$ as an interesting target for pharmacotherapeutic strategies in the prevention of neuronal damage in $\mathrm{AD}$ and related neuroinflammatory diseases.

\section{Part II. Presented by Francisco Di Virgilio}

Ceruti S, Viganò F, Boda E, Ferrario S, Magni G, Boccazzi M, Rosa P, Buffo A, Abbracchio MP (2011) Expression of the New P2Y-Like Receptor GPR17 During Oligodendrocyte Precursor Cell Maturation Regulates Sensitivity to ATPInduced Death. Glia 59:363-378; DOI 10.1002/glia.21107

\section{Article summary}

The P2Y-like receptor GPR17 has been recently identified as an interesting modulator of oligodendrocyte differentiation during maturation and in myelinating disorders. In the present study, the expression and function of GPR17 in primary cultures of astrocytes and oligodendrocyte precursor cells (OPCs) were investigated. It has been shown that GPR17 expression was markedly influenced by culture conditions: (1) In the presence of growth factors (GFs), no significant GPR17 expression was observed, whereas (2) in the absence of GFs, the receptor was found in morphologically more mature OPCs. After the shift of (1) into (3), a differentiating medium, a time-dependent increase in the number of GPR17-positive cells occurred, in the totality of O4-expressing cells. The shift from (2) into (3) resulted in GPR17 expression only in a subpopulation of O4. Finally, under both protocols, the appearance of more mature cells (CNPase or MBP positive) was associated with a progressive loss of GPR17.

When cultures were treated with (4) adenine nucleotides, mimicking the increase in extracellular nucleotides observed under pathophysiological conditions, GPR17 expression sensitized cells to ATP-induced cytotoxicity, whereas activation with (5) uracil nucleotides promoted their differentiation towards a more mature phenotype. 
In summary, the present in vitro data indicate that GPR17 expression in OPCs depends upon the presence of GFs and highlight its major role in oligodendrocyte maturation. Furthermore, GPR17 expression was interpreted as a "danger signal" in the presence of high extracellular ATP concentrations, suggesting an important role of the receptor in brain disorders.

Fumagalli M, Daniele S, Lecca D, Lee PR, Parravicini C, Fields RD, Rosa P, Antonucci F, Verderio C, Trincavelli ML, Bramanti P, Martini C, Abbracchio MP (2011) Phenotypic changes, signaling pathway and functional correlates of GPR17-expressing neural precursor cells during oligodendrocyte differentiation. JBC, 2011, in press; DOI 10.1074/jbc.M110.162867. Published online on Jan 5, 2011

\section{Article summary}

In the present study, purified OPCs were used to define GPR17 expression during the differentiation process. It was found that the receptor gradually increases in very early differentiation stages and is progressively turned down in morphologically mature myelinating cells. Furthermore, GPR17 was expressed by two subsets of slowly proliferating OPCs that co-express the proteoglycan NG2: (1) morphologically-immature cells expressing early oligodendrocyte-specific proteins (e.g. Olig2, PDGF R- $\alpha$ ) and (2) ramified, still immature preoligodendrocytes expressing more mature oligodenrocytespecific proteins (e.g. O4, O1).

Moreover, for the first time in a native cell system, the signalling pathway of GPR17 was characterized. Activation by its endogenous ligands resulted in a potent inhibition of intracellular cAMP formation. This effect was counteracted by GPR17 antagonists and receptor silencing with siRNAs.

In summary, these data highlight GPR17 as a new marker that specifically labels distinct early stages of OPC differentiation in the developing and mature CNS. The characterization of the signalling pathway of native GPR17 and its relation to OPC differentiation clearly establishes a mechanistic role for this receptor and its endogenous ligands under physiological conditions and in CNS demyelinating disorders, e.g. multiple sclerosis (MS).

\section{Combined commentary}

The pathological changes of MS in human CNS, contributing to neurological disabilities, include inflammation, demyelination, oligodendrocyte death and axonal degeneration. During the early stages of MS, extensive remyelination can occur and oligodendrocytes have been indeed detected in remyelinating MS lesions. Therefore, the endogenous reparative potential of OPCs, as a possible source of newly generated cells under physiological and pathophysiological conditions, are of very special interest. The data of these two papers highlight new important knowledge that may be helpful for the identification of critical regulators and the understanding of the mechanisms instructing or limiting the maturation of OPCs. Specifically, these recent data by the Laboratory of Molecular and Cellular Pharmacology of Purinergic Transmission in Milan demonstrate GPR17 expression by OPCs in adult brain and its "double role" as an interesting modulator of oligodendrocyte function.

After injury or inflammation, not only adenine nucleotides (see above) but also uracil nucleotides (i.e. UDP, UDPsugars) and cysteinyl leukotrienes (i.e. LTC4, LTD4), the putative endogenous agonists of the GPR17, are massively released at the site of injury. These ligands may contribute to initiating repair responses. Thus, in addition to the already well-known modulating role of GFs on the maturation of OPCs, uracil nucleotides can possibly work in synergy with GFs on adult progenitor cells to promote their maturation to fully functional cells.

In this respect, the data of Ceruti et al. very convincingly demonstrate the importance of culture conditions for the proliferation of OPCs, their morphology, receptor expression and response to exogenous stimuli. GFs (EGF, bFGF) may keep OPCs in a less-differentiated stage by restraining GPR17 expression, whereas under permissive conditions, GPR17 contributes to OPC differentiation. Furthermore, the more mature GPR17 positive cells, detected under growth conditions without GFs, appeared less prone to react to the exposure to differentiation triggers. The effects of external stimuli (e.g. nucleotides) on astrocytes to induce astrogliosis are well characterized. Remarkably, in the used mixed cultures, no GPR17 expression on GFAP-positive astrocytes (conform to in vivo data of the group; Lecca et al. 2008, PLoS One, 3(10):e3579) and only a slight astroglial reaction under the different treatment conditions were observed.

A main topic of this study is the investigation of the endogenous initial repair response of OPCs in relation to changes in GPR17 expression. The number of GPR17positive cells was significantly reduced in the presence of ATP, whose levels are known to increase to toxic concentrations after lesions. It is hypothesized that the different extent of receptor expression might have influenced cell susceptibility to ATP-induced cell death. Interestingly, ATP has been shown to act as an antagonist at recombinant GPR17 (Pugliese et al. 2009, Am J Physiol Cell Physiol, 297:C1028-C1040), suggesting that under disease conditions, the excessively released ATP, by binding to GPR17, may counteract the trophic effects 
mediated by uracil nucleotides under physiological conditions. In summary, besides demonstrating the specific role of GPR17 in the maturation of OPCs under physiological conditions, these data underline the potential role of GPR17 in the massive cell death occurring during pathophysiological conditions-two visions of one receptor.

In the paper of Fumagalli et al., the authors went further into this topic and focused on phenotypic and molecular characteristics of GPR17 and its transduction system. They report changes in receptor expression of rat primary OPCs continuously differentiating to myelinforming oligodendrocytes. Besides, the use of typical pre-oligodendroglial markers (e.g. O4) and markers of mature myelinating oligodendrocytes (e.g. MBP), especially the expression of the NG2, was studied. All results are illustrated in a helpful overview and indicate clear the GPR17 expression as a function of the OPC maturation, restricted to cells at early differentiation stages. In this relationship, the special expression behaviour of GPR17/NG2-positive cells should be emphasized. Data of studies in human MS tissue suggest functional subpopulations of NG2-positive cells, existing in the MS lesions (Chan et al. 2000, J Neurosci, 20:6404-6412). Therefore, the knowledge about GPR17/NG2 co-expression in OPCs could be helpful for further understanding the mechanisms at the basis of remyelination processes.

For the first time, this paper also shows that at variance from recombinant GPR17, activation of native GPR17 by the endogenous ligands did not affect intracellular calcium levels but resulted in marked inhibition of cAMP formation (conform to data of the group using recombinant human and rodent GPR17; Ciana et al. 2006, EMBO J, 25:4615-4627).
Additionally, experiments with the specific antagonists and receptor silencing studies confirmed that these effects are selectively due to stimulation of GPR17. Furthermore, the used agonists promoted and the antagonists/receptor silencing inhibited OPC maturation in vitro.

In their final hypothesis, the authors highlight, among others, two important stages of GPR17 expression in OPC differentiation. In detail, they accent to (1) an early differentiation stage, where the receptor may keep cells in an immature state, necessary to prepare them for myelination and (2) a later critical stage of differentiation, at which the receptor is downregulated, a step that seems necessary to allow cells to complete their maturation. Therefore, GPR17 is a "key timer" in the regulation of oligodendrocyte differentiation and terminal maturation, and might serve as a potential therapeutical target for myelin repair in CNS demyelinating diseases, such as MS.
About the author
Heike Franke is associated professor at the Rudolf Boehm Institute of Pharmacology and Toxicology at the University of Leipzig, Germany. Her research interest relates to trophic and toxic actions of ATP in the CNS. At present, her focus is (1) the characterization and modulation of astrogliosis; (2) the study of receptor expression and purinergic signal- ling after acute brain injury (trauma, ischemia), (3) growth- promoting effects of $\mathrm{P} 2$ receptors and selected compounds in organotypic slice co-cultures of the dopaminergic system and (4) neurogenesis. 